\title{
Multiparametric MRI/TRUS Fusion Prostate Biopsy: Advantages of a Transperineal Approach
}

\author{
PIETRO PEPE ${ }^{1}$, ANTONIO GARUFI ${ }^{2}$, GIAN DOMENICO PRIOLO ${ }^{2}$ and MICHELE PENNISI ${ }^{1}$ \\ ${ }^{1}$ Urology Unit - Cannizzaro Hospital, Catania, Italy; \\ ${ }^{2}$ Imaging Department - Cannizzaro Hospital, Catania, Italy
}

\begin{abstract}
Background/Aim: To evaluate the detection rate for clinically-significant prostate cancer (PCa) of transperineal (TP) vs. transrectal (TR) multiparametric MRI/TRUS (magnetic resonance imaging/transrectal ultrasound) fusion targeted-biopsy. Patients and Methods: From January 2016 to December 2016, 150 men underwent repeat saturation transperineal prostate biopsy (SPBx; median 30 cores) combined with targeted mpMRI/TRUS TR and TP fusion biopsies (4 cores for each procedure) of suspicious MRI lesions (PI-RADS 3/5). Results: Overall, in 55/150 (36.6\%) men a clinically-significant PCa was found and in 49 (89.1\%) of them mpMRI was positive. SPBx, mpMRI/TRUS TR and TP fusion targeted-biopsy diagnosed 52 (94.5\%), 43 (78.1\%) and 49 (89.1\%) PCa, respectively; TR fusion biopsy missed 8 (53.3\%) while TP missed 2 (13.3\%) cancers of the anterior zone. Conclusion: Multiparametric MRI/TRUS TP in comparison to TR fusion biopsy detected a greater percentage of small but clinically significant PCa of the anterior zone (86.7\% vs. $46.7 \% ; p=0.0001$ ).
\end{abstract}

Prostate cancer (PCa) is the most frequent tumor diagnosed in elder men with about 1 million biopsies performed in the United States annualy (1). The rate of overdiagnosis in men enrolled in screening protocols is equal to $50 \%$ of the cases (2). Moreover, the transrectal biopsy approach is associated with an increased risk of infection with an estimated hospital admission and sepsis equal to $2.5 \%$ (3) and $3.5 \%$ (4), respectively. Therefore, the main goal is to reduce the number of unnecessary biopsies and diagnose only clinically significant $\mathrm{PCa}$. In this respect, multiparametric magnetic resonance imaging (mpMRI) combined with TRUS (transrectal ultrasound) fusion targeted biopsy has improved the accuracy

Correspondence to: Dr. Pietro Pepe, Urology Unit - Cannizzaro Hospital, Via Messina 829, Catania, Italy. Tel: +39 957263285, Fax: +39 957263259, e-mail: piepepe@ hotmail.com

Key Words: Prostate cancer, multiparametric MRI, transrectal fusion biopsy, transperineal fusion biopsy, saturation prostate biopsy. of standard biopsy schemes in detecting clinically-significant prostate cancer ( $\mathrm{PCa}$ ), especially, in case of a repeat biopsy (57) and in the reevaluation of men enrolled in active surveillance (AS) programs (8-10). Although the accuracy of mpMRI/fusion targeted biopsy has been evaluated in a lot of series, very few papers have compared the detection rate for $\mathrm{PCa}$ or/and complications of the different MRI/TRUS fusion platforms in the same population (11-14). On the other hand, the standard transperineal biopsy approach in comparison with the transrectal procedure has demostrated a higher accuracy in diagnosing PCa located in the anterior zone of the gland (15) resetting the risk of sepsis (16).

In this report, the detection rate for clinically-significant PCa (17) performing transperineal (TP) vs. transrectal (TR) mpMRI/TRUS fusion targeted-biopsy has been prospectively evaluated in men submitted to repeat prostate biopsy.

\section{Patients and Methods}

From January 2016 to December 2016150 men (median age 62 years; range $=47-78$ years) with negative digital rectal examination underwent repeat saturation transperineal prostate biopsy (SPBx) for the persistent suspicion of cancer (increasing or persistent elevated PSA values). All the patients 10 days before SPBx underwent pelvic mpMRI; SPBx (median 30 cores; range=28-34 cores) was performed transperineally by a GE Logiq P6 ecograph (General Electric; Milwaukee, WI) supplied with a bi-planar trans-rectal probe (5-7.5 MHz) using a tru-cut 18 gauge needle (Bard; Covington, GA) under sedation and antibiotic prophylaxis (18). All mpMRI examinations were performed using a 3.0 Tesla scanner, (ACHIEVA 3T; Philips Healthcare Best, the Netherlands) equipped with surface 16 channels phased-array coil placed around the pelvic area with the patient in the supine position; multi-planar turbo spinecho T2-weighted (T2W), axial diffusion weighted imaging (DWI), axial dynamic contrast enhanced (DCE) and spectroscopy were performed for each patient. The mpMRI lesions characterized by a PI-RADS (Prostate Imaging-Reporting and Data System) score of $3 / 5$ were considered suspicious for cancer $(5,7)$; two radiologists blinded to pre-imaging clinical parameters evaluated the mpMRI data separately and independently. Informed consent was obtained from all individual participants included in the study. In the presence of mpMRI lesions suggestive of cancer, mpMRI/TRUS TR and TP fusion guided-biopsies (4 cores for each procedure) were 
Table I. Biopsy histological findings in the 55 men with clinically significant prostate cancer (PCa) diagnosed by mpMRI/TRUS transperineal (TP) vs. transrectal (TR) fusion biopsy

\begin{tabular}{lcc}
\hline Biopsy histological findings and mpMRI results & TRUS/mpMRI TR fusion targeted biopsy & TRUS/mpMRI TP fusion targeted biopsy \\
\hline mpMRI: true positive & $49(89.1 \%)$ & $49(89.1 \%)$ \\
mpMRI: false negative & $6(10.9 \%)$ & $6(10.9 \%)$ \\
mpMRI: false positive & $16(22.5 \%)$ & $16(22.5 \%)$ \\
Clinically significant PCa* (overall: 55cases) & $43(78.1 \%)$ & $49(89.1 \%)$ \\
Anterior zone PCa (overall: 15 cases) & $7(46.7 \%)$ & $13(86.7 \%)$ \\
PCa of peripheric zone (overall: 40 cases) & $36(90 \%)$ & $36(90 \%)$ \\
Gleason score & & 29 \\
$3+4$ & 25 & 17 \\
$4+3$ & 15 & 2 \\
$4+4$ & 2 & 1 \\
$5+4$ & $12(9-20)$ & $8(5-20)$ \\
Diameter of mpMRI lesion** (range: millimeter) & $2(1-4)$ & $3.2(1-4)$ \\
Number of positive cores** (range) & & \\
\hline
\end{tabular}

TRUS: Transrectal ultrasound; mpMRI: multiparametric magnetic resonance imaging; *Clinically significant PCa (prostate cancer): $>2$ positive cores with Gleason score $>6$; **Median.

added to SPBx using a GE Logiq E9 and Hitachi 70 Arietta ecograph (Hitachi Medico, Chiba, Japan), respectively $(12,19)$. The GE Logiq E9 and Hitachi Arietta 70 platforms allowed processing of a software-based rigid registration of pelvic mpMRI and TRUS (end-fire probe and biplanar probe, respectively) by the use of a fusion device; moreover, an electromagnetic tracking system showed the needle localization (Figure 1).

The accuracy of TP $v s$. TR mpMRI/TRUS fusion targeted biopsy in diagnosing clinically significant PCa (Gleason score $>6$ and/or more than 2 positive cores) were prospectively evaluated (20) and compared with SPBx results. For statistical analysis the Student's T test was used; a $p$-value $<0.05$ was considered statistically significant.

\section{Results}

All patients had a negative TRUS (median prostate weight $45 \mathrm{~g}$; range=25-95g) and median PSA was $9.2 \mathrm{ng} / \mathrm{ml}$ (range $=4.5-31 \mathrm{ng} / \mathrm{mL}$ ); mpMRI showed a suspicious lesion (PI-RADS 3/5) in $71(47.3 \%$ ) cases. Overall, in 55/150 $(36.6 \%)$ men a clinically significant $\mathrm{PCa}$ was found and in $49(89.1 \%)$ of them mpMRI was positive (PI-RADS $3=18$ cases; PI-RADS $4=6$ cases; PI-RADS $5=15$ cases); in the remaining $12(8 \%)$ and $78(52 \%)$ patients a cancer at risk for indolent disease and a normal parenchyma was detected, respectively. In 55 men with clinically significant PCa 392 targeted biopsies (196 cores for TR and TP fusion procedure) were performed in the mpMRI suspicious lesions; in 40 $(72.7 \%)$ and $15(27.3 \%)$ cases, $\mathrm{PCa}$ was found in the peripheric and anterior zone of the gland, respectively. None had significant complications from prostate biopsy that needed hospital admission; moreover, the mpMRI procedure was well tolerated and successfully performed in all cases.

The biopsy quantitative histology (i.e., number of positive cores, greatest percentage of cancer "GPC"), Gleason score and mpMRI findings in the presence of PCa are listed in Table I.
SPBx, mpMRI/TRUS TR fusion and TP fusion targeted biopsy diagnosed $52(94.5 \%), 43(78.1 \%)$ and $49(89.1 \%)$ clinicallysignificant PCa, respectively. SPBx, TR fusion and TP targeted biopsy missed $3(20 \%), 8(53.3 \%)$ and $2(13.3 \%)$ cancers of the anterior zone and $0,4(10 \%)$ and $4(10 \%)$ of the peripheric gland, respectively. The TR fusion approach missed 6/49 clinically significant PCa that were diagnosed by TP fusion biopsy; in detail, all the PCa were located only in the anterior zone of the gland and were provided of a GS, GPC and mpMRI diameter equal to 7,50\% and 8 millimeter, respectively.

The detection rate of cancer for each core performing SPBx, TR and TP targeted biopsy was $12 \%, 30 \%$ and 55\%, respectively. Sensitivity, specificity, positive predictive value, negative predictive value (NPV) and diagnostic accuracy of TP-fusion $v s$. TR-fusion targeted biopsy were equal to 89.1 vs. $78.2 \%, 86.3$ vs. $77.2 \%, 69$ vs. $60.5 \%, 94$ vs. $88.7 \%, 87.2$ vs. $84.2 \%$, respectively.

\section{Discussion}

The improvement of diagnostic imaging by mpMRI has allowed to perform targeted biopsies of suspicious areas increasing the accuracy in the diagnosis of clinicallysignificant PCa $(21,25)$ resulting in predictive of definitive Gleason score with a higher detection rate of cancer for each core in comparison to standard prostate biopsy schemes. The detection rate for PCa of mpMRI is between $39 \%$ and $59 \%$ $(26,27)$ with an incidence of cancer located only in the anterior zone equal to $20 \%(16,28)$. Although mpMRI is strongly recommended in men who are candidates to repeat biopsy or enrolled in AS protocols (8-11), still today, extended or SPBx should always be combined with mpMRI/TRUS fusion biopsy because of the false negative 


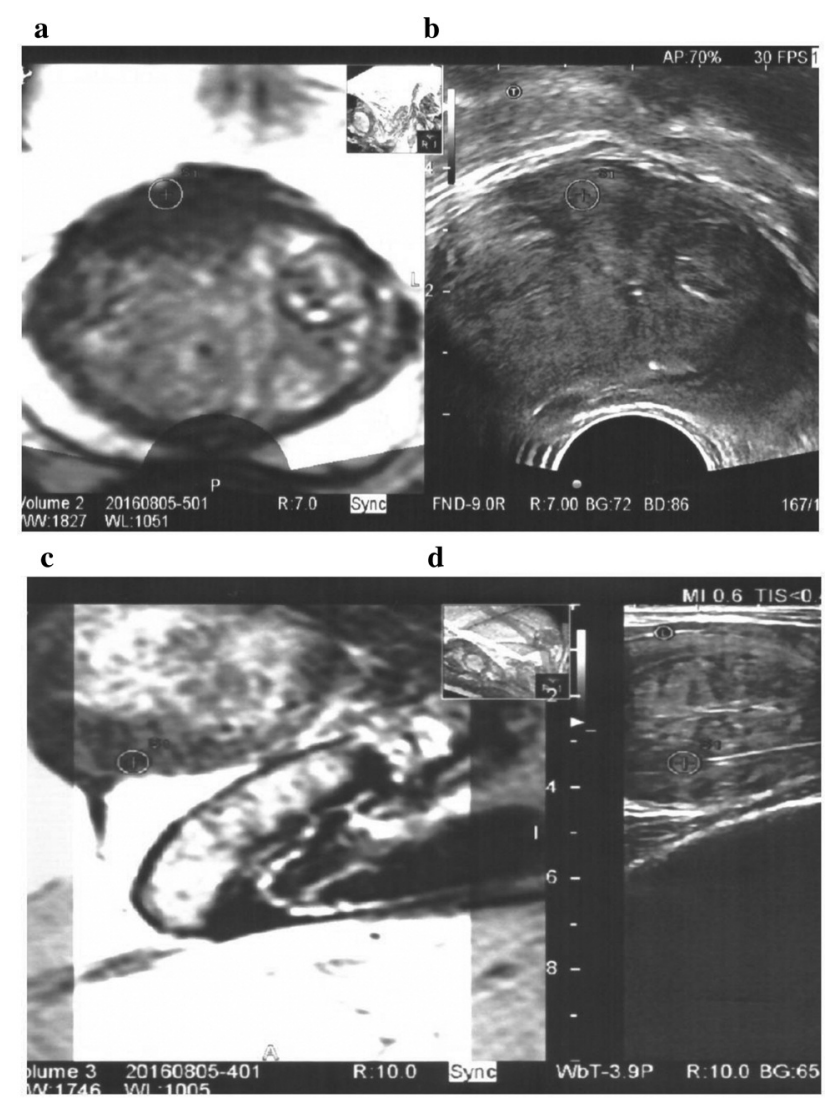

Figure 1. Multiparametric MRI/TRUS fusion image (caliper) of a prostate cancer located in the anterior zone (a: axial T2W-PI-RADS 4; $b$ : axial TRUS). Transperineal mpMRI/TRUS fusion targeted biopsy of the suspicious lesion (c: sagittal mpMRI; d: sagittal TRUS during fusion targeted biopsy).

rate of mpMRI (15-20\% of PCa with low volume and GS $>7$ ) and the variable diagnostic accuracy reported using the different mpMRI/TRUS fusion biopsy platform (12, 22-24). The targeted biopsy of mpMRI suspicious areas could be performed using "in-bore" mpMRI-guidance, real-time mpMRI/TRUS imaging fusion or by performing cognitive mpMRI/TRUS biopsies (29, 30). Recently, many papers have demonstrated a higher accuracy in favour of the fusion technique; conversely, few data have been reported regarding the accuracy of TR $v s$. TP mpMRI/TRUS fusion approach in diagnosing clinically significant PCa $(12,13)$. In this respect, standard TP and TR prostate biopsies are provided of a superimposable detection rate for $\mathrm{PCa}$ (4-17), but, at the same time, the transperineal approach allows to easily and better reach the anterior zone of the gland (15) resetting the risk of sepsis (16).

In our series, the detection rate for clinically significant PCa was lower performing the TR $(78.1 \%) v s$. TP $(89.1 \%)$ fusion approach. In detail, the TP targeted biopsies diagnosed more PCa $(86.7 \%)$ located in the anterior zone of the prostate in comparison with TR approach $(46.7 \% ; p=0.0001)$ and SPBx $(80 \%)$. Moreover, clinically significant PCa detected by TR mpMRI/TRUS fusion biopsy had a greater mpMRI lesion diameter in comparison with the TP approach; $12 \mathrm{vs}$. 8 millimeter, respectively. In definitive, TP and TR fusion biopsy diagnosed the same percentage of PCa located in the peripheric zone of the gland (90\% of the cases), conversely, the TP fusion targeted approach allowed to diagnose a greater number of clinically significant PCa of the anterior zone (6 out 15 equal to $40 \%$ of the cases) charaterized by a small volume showing a NPV for PCa equal to $94 \%$. These data could be useful in the planning of a mpMRI/TRUS fusion targeted biopsy of small mpMRI lesions located in the anterior prostate near the pubic bone.

Regarding our results certain considerations should be made. Firstly, an "in-bore" approach could improve the detection rate for $\mathrm{PCa}$; secondly, biopsy histological findings should be compared with the whole prostate specimen to evaluate the false positive mpMRI results (about $20 \%$ of cases). Finally, a greater number of patiens submitted to TR vs. TP mpMRI/TRUS fusion biopsy should be compared.

In conclusion, although SPBx diagnosed the majority of clinically-significant PCa (94.5\% of the cases), mpMRI/TRUS TP in comparison with TR fusion biopsy detected a greater percentage of small, but clinically-significant PCa of the anterior zone ( $86.7 \%$ vs. $46.7 \%$; $p=0.0001)$.

\section{References}

1 Loeb B, Carter HB, Berndt SI, Ricker W and Schaeffer EM: Complications after prostate biopsy: data from SEE-Medicare. J Urol 186: 1830-1844, 2011.

2 Schröder FH, Hugosson J, Roobol MJ, Tammela TL, Ciatto S, Nelen V, Kwiatkowski M, Lujan M, Lilja H, Zappa M, Denis LJ, Recker F, Páez A, Määttänen L, Bangma CH, Aus G, Carlsson S, Villers A, Rebillard X, van der Kwast T, Kujala PM, Blijenberg BG, Stenman UH, Huber A, Taari K, Hakama M, Moss SM, de Koning HJ and Auvinen A; ERSPC Investigators: Prostate-cancer mortality at 11 years of follow-up. N Engl J Med 366: 981-990, 2012.

3 Pinkhasov GI, Lin YK, Palmerola R, Smith P, Mahon F, Kaag MG, Dagen JE, Harpster LE, Reese CT and Raman JD: Complications following prostate needle biopsy requiring or emergency department visits- experience from 1000 consecutive cases. BJU Int 110: 369-374, 2012.

4 Ehdaie B, Vertosick E, Spaliviero M, Giallo-Uvino A, Taur Y, O'Sullivan M, Livingston J, Sogani P, Eastham J, Scardino P and Touijer K: The impact of repeat biopsies on infectious complications in men with prostate cancer on active surveillance. J Urol 191: 660-664, 2014.

5 Pepe P, Garufi A, Priolo G and Pennisi M: Can 3 Tesla pelvic phase-array MRI avoid unnecessary repeat prostate biopsy in patients with PSA below $10 \mathrm{ng} / \mathrm{ml}$ ? Clin Genitourin Cancer 13: e27-30, 2015. 
6 Roethke MC, Kuru TH, Schultze S, Tichy D, Kopp-Schneider A, Fenchel M, Schlemmer HP and Hadaschik BA: Evaluation of the ESUR PI-RADS scoring system for multiparametric MRI of the prostate with targeted MR/TRUS fusion-guided biopsy at 3.0 Tesla. Eur Radiol 24: 344-352, 2014.

7 Pepe P, Garufi A, Priolo G, Candiano G, Pietropaolo F, Pennisi M, Fraggetta F and Aragona F: Prostate cancer detection at repeat biopsy biopsy: can pelvic phased-array multiparametric MRI replace saturation biopsy? Anticancer Res 33: 1195-1199, 2013.

8 Hoeks CM, Somford DM, van Oort IM, Vergunst H, Oddens JR, Smits GA, Roobol MJ, Bul M, Hambrock T, Witjes JA, Fütterer JJ, Hulsbergen-van de Kaa CA and Barentsz JO: Value of 3-T multiparametric magnetic resonance imaging and magnetic resonance-guided biopsy for early risk restratification in active surveillance of low-risk prostate cancer: a prospective multicenter cohort study. Invest Radiol 49: 165-172, 2014.

9 Fascelli M, George AK, Frye T, Turkbey B, Choyke PL and Pinto PA: The role of MRI in active surveillance for prostate cancer. Curr Urol Rep 16(6): 42, 2015.

10 Barrett $\mathrm{T}$ and Haider MA: The emerging role of MRI in prostate cancer active surveillance and ongoing challenges. AJR Am J Roentgenol 208(1): 131-139, 2017.

11 Westhoff N, Siegel FP, Hausmann D, Polednik M, von Hardenberg J, Michel MS and Ritter M: Precision of MRI/ultrasound-fusion biopsy in prostate cancer diagnosis: an ex vivo comparison of alternative biopsy techniques on prostate phantoms. World J Urol Nov 9, 2016.

12 Pepe P, Garufi A, Priolo G and Pennisi M: Transperineal versus transrectal MRI/TRUS fusion targeted biopsy: detection rate of clinically significant prostate cancer. Clin Genitourin Cancer 15(1): e33-e36, 2016.

13 Kongnyuy M, George AK, Rastinehad AR and Pinto PA: Magnetic resonance imaging-ultrasound fusion-guided prostate biopsy: review of technology, techniques, and outcomes. Curr Urol Rep 17(4): 32, 2016.

14 Rastinehad AR, Abboud SF, George AK, Frye TP, Ho R, Chelluri R, Fascelli M, Shih J, Villani R, Ben-Levi E, Yaskiv O, Turkbey B, Choyke PL, Merino MJ, Wood BJ and Pinto PA: Reproducibility of multiparametric MRI and fusion-guided prostate biopsy: multi-institutional external validation by a propensity score matched cohort. J Urol 195: 1737-1783, 2016.

15 Pepe $\mathrm{P}$ and Aragona F: Morbidity following transperineal prostate biopsy in 3,000 patients submitted to $12 \mathrm{vs} .18 \mathrm{vs}$. more than 24 needle cores. Urology 81: 1142-1146, 2013.

16 Pepe P, Dibenedetto G, Pennisi M, Fraggetta F, Colecchia M and Aragona F: Detection rate of anterior prostate cancer in 226 patients submitted to initial and repeat transperineal biopsy. Urol Int 93: 189-192, 2014.

17 Valerio M, Anele C, Bott SR, Charman SC, van der Meulen J, ElMahallawi H, Emara AM, Freeman A, Jameson C, Hindley RG, Montgomery BS, Singh PB, Ahmed HU and Emberton M: The prevalence of clinically significant prostate cancer according to commonly used histological thresholds in men undergoing template prostate mapping bopsies. J Urol 195: 1403-1408, 2016.

18 Pepe P and Aragona F: Saturation prostate needle biopsy and prostate cancer detection at initial and repeat evaluation. Urology 70: 1131-1135, 2007

19 Fandella A, Scattoni V, Galosi A, Pepe P, Fiorentino M, Gaudiano C, Giampaoli M, Gunelli R, Martino P, Montanaro V,
Montironi R, Pierangeli T, Stabile A and Bertaccini A: Italian prostate biopsies group. Anticancer Res 37: 413-424, 2017.

20 Epstein J, Walsh P and Carmichael M: Pathological and clinical findings to predict tumor extent of non palpable (stage T1c) prostate cancer. JAMA 271: 368-374, 1994.

21 Sonn GA, Natarajan S, Margolis DJ, MacAiran M, Lieu P, Huang J, Dorey FJ and Marks LS: Targeted biopsy in the detection of prostate cancer using an office based magnetic resonance ultrasound fusion device. J Urol 189: 86-92, 2013.

22 Filson CP, Natarajan S, Margolis DJ, Huang J, Lieu P, Dorey FJ, Reiter RE and Marks LS: Prostate cancer detection with magnetic resonance-ultrasound fusion biopsy: The role of systematic and targeted biopsies. Cancer 122: 884-892, 2016.

23 Recabal P, Assel M, Sjoberg DD, Lee D, Laudone VP, Touijer K, Eastham JA, Vargas HA, Coleman J and Ehdaie B: The Efficacy of multiparametric magnetic resonance imaging and magnetic resonance imaging targeted biopsy in risk classification for patients with prostate cancer on active surveillance. J Urol 196: 374-381, 2016.

24 Pepe P, Garufi A, Priolo G and Pennisi M: Can MRI/TRUS fusion targeted biopsy replace saturation prostate biopsy in the re-evaluation of men in active surveillance? World J Urol 34: 1249-1453, 2016.

25 Hansen NL, Kesch C, Barrett T, Koo B, Radtke JP, Bonekamp D, Schlemmer HP, Warren AY, Wieczorek K, Hohenfellner M, Kastner C and Hadaschik B: Multicentre evaluation of target and systematic biopsies using Magnetic Resonance and Ultrasound Image-Fusion guided Transperineal Prostate Biopsy in patients with a previous negative biopsy. BJU Int Nov 10, 2016. doi: 10.1111/bju.13711. [Epub ahead of print]

26 Franiel T, Stephan C, Erbersdobler A, Dietz E, Maxeiner A, Hell N, Huppertz A, Miller K, Strecker R and Hamm B: Areas suspicious for prostate cancer: MR-guided biopsy in patients with at least on US-guided biopsy with a negative finding. Multiparametric MR imaging for detection and biopsy planning. Radiology 259: 162-72, 2011.

27 Hambrock T, Somford DM, Hoeks C, Bouwense SA, Huisman H, Yakar D, van Oort IM, Witjes JA, Fütterer JJ and Barentsz JO: Magnetic resonance imaging-guided prostate biopsy in men with repeat negative biopsies and increased prostate-specific antigen. J Urol 185: 520-527, 2010.

28 Volkin D, Turkbey B, Hoang AN, Rais-Bahrami S, Yerram N, Walton-Diaz A, Nix JW, Wood BJ, Choyke PL and Pinto PA: Multiparametric magnetic resonance imaging (MRI) and subsequent MRI/ultrasonography fusion-guided biopsy increase the detection of anteriorly located prostate cancers. BJU Int 114(6b): E43-9, 2014

29 Cool DW, Zhang X, Romagnoli C, Izawa JI, Romano WM and Fenster A: Evaluation of MRI-TRUS fusion versus cognitive registration accuracy for MRI-targeted, TRUS-guided prostatebiopsy. Am J Roentgenol 204: 83-91, 2015.

30 Schimmöller L, Quentin M, Blondin D, Dietzel F, Hiester A, Schleich C, Thomas C, Rabenalt R, Gabbert HE, Albers P, Antoch G and Arsov C: Targeted MRI-guided prostate biopsy: are two biopsy cores per MRI-lesion required? Eur Radiol 26: 3858-3864, 2016.

Received April 28, 2017

Revised May 13, 2017

Accepted May 15, 2017 